\section{Trace elements in metabolism}

Trace Elements and Iron in Human Metabolism. By A.S. Prasad. Pp. 380. (Plenum: New York, 1979). Available in UK, Middle East and Africa from Wiley (Chichester, UK) at $£ 17.50 ; \$ 35$.

IF a monograph is defined as a systematic and exhaustive examination of a particular subject, then this book, contrary to the statement in the Foreward, cannot be so described. It contains information about not only the wellestablished (chromium, copper, fluorine, iodine, manganese and selenium) and newer (nickel, vanadium, silicon and tin) trace elements, but also magnesium, iron and certain toxic metals. This last section is highly selective in its choice of metals (cadmium, lead and mercury) and seems quite out of place in the present volume; the brief chapters are superficial and contain statements that are either incorrect, or likely to mislead. Thus, for example: "hypertensive subjects have abnormally high concentrations of renal cadmium" (p354); "A similar protective effect of excess zinc against lead toxicity was noted in young horses" (p365); and, "The physiocochemical classification of mercury does not correlate well with its toxic properties" (p371).

Even the review of the field of essential metals is an enormous task for a single author, and although Dr Prasad has produced an informative summary, inevitably many of the recent developments have been omitted. The chapter on chromium (confined essentially to the glucose-tolerance factor), selenium (devoted largely to glutathione peroxidase) and the newer trace elements, for example, contain only 2,2 and 1 reference(s), respectively, to work published later than 1975, whilst

- The Sense of Order: A Study in the Psychology of Decorative Art (Phaidon: Oxford, 1979), by E.H. Gombrich (for review, see Nature, 279, 653; 1979), has been published in the United States by Cornell University Press (Ithaca, New York) at $\$ 38.50$.

- The Menace of Atomic Energy (W.W. Norton: New York, 1977), by Ralph Nader and John Abotts (for review, see Nature, 274, $95 ; 1978$ ) has now been published in the UK by Melbourne House (London) at $£ 7.95$.

- Rutherford and Physics at the Turn of the Century, edited by M. Bunge and W.R. Shea (for review, see Nature, 279, 741; 1979), was published by Neale Watson Academic (New York) and Dawson (Folkestone, UK), and not as stated. in the chapter on iron (a detailed, 60-page, review) $94 \%$ of the 428 references are to publications before 1971. Also the references to published work are perhaps inevitably for such a large subject, rather selective. Nevertheless, the book provides a clear account, not by any means exhaustive, of certain aspects of the action of trace elements aiming to link molecular biology, biological and animal experimentation with human nutrition and clinical experience. This is a highly commendable aim and the book can be recommended to provide such a broad view which, if depth is required, will require supplementation with further reading. The individual chapters on copper, iron and zinc, in particular, are extremely thorough and informative.

The very readable text is, in some cases, rather repetitious largely due to the subdivisions in each chapter, such that the experimental work in animals is often separated from observations in man. Furthermore, the considerations of the functions and mechanism of action of the

\section{Electromagnetic wave propagation}

Methods in Electromagnetic Wave Propagation. By D.S. Jones. Pp. 887. (Clarendon/Oxford University Press: Oxford, 1979.) £22.50.

THE field of electromagnetism has, as the author claims, undergone considerable development in recent years. Motivated by such fields as satellite communications, radar and geophysical prospecting the search for better methods of solution to important problems in the areas of electromagnetic wave propagation and scattering has intensified. Existing classical methods of applied analysis have been extended, complemented or indeed supplanted by new numero/theoretic methods based upon modern applicable mathematics. Professor Jones, himself a leading researcher in the field, is to be congratulated on presenting these methods in a systematic manner and critically reviewing their application.

The book is very large, containing over 800 pages in 9 chapters. The first chapter is introductory and contains the mathematical features used in the remainder of the book. The problems of propagation in waveguides are discussed in chapters 2,3 and 4 . Solutions are found by finite differences (chapter 2) and by variational methods (chapter 4). As these problems can often be reduced to finding eigenvalues of operators, this is described in some detail in chapter 3 . The implementation of variational methods to provide numerical bounds on approximate solutions is the main concern of chapter 5 . trace elements are somehow diluted by sections on toxicity and therapeutic measures. The book is well produced, but for such a wide general coverage of the subject, a more detailed index might have been an advantage.

With the provisos mentioned above, this book can be recommended for those who wish to quickly obtain a general view of the biology of trace elements with particular reference to man. Contrary to the title, the volume is not restricted to human metabolism, but at least in certain sections also deals extensively with studies in experimental and domestic animals. Thus, it covers much but not all, of the ground already surveyed by Underwood in the fourth edition (1977) of his book Trace Elements in Human and Animal Nutrition (Academic; New York, San Francisco and London).

\section{N. Aldridge}

M. Webb

N. Aldridge and M. Webb are at the MRC Toxicology Unit, Carshalton, UK.

The advent of the digital computer has transformed the antenna design field and classically 'difficult' problems are now amenable to numerical analysis. Both wire and solid antennas are treated in chapter 6 where integral equations of various types are studied from analytical and numerical points of view. Guidance on methods for dealing with transient phenomena is given in chapter 7 .

The most exciting material in the book for the reviewer is in chapter 8 where the geometric theory of diffraction (GTD) is developed from first principles. Very few authoritative accounts of this subject are available and the author has devoted one quarter of his book to it. The use of GTD in the design of reflector antennas has become increasingly important in recent years. Finally the problems of inverse scattering, that is, describing the source knowing its scattered field, is discussed in chapter 9 . Nearly 400 references are listed, although few appear for 1977 and none afterwards.

It is perhaps churlish to mention omissions in a book of this length but no space has been found for recent work on general reflector synthesis involving Monge-Ampere equations or the use of complex coordinates and stereographic projection in geometrical optics.

The quality of the material and the clarity of its exposition makes this an important reference book for research workers and lecturers (there are numerous exercises) in the electromagnetic field.

B.S. Westcott

B.S. Westcott is Senior Lecturer in Applied Mathematics in the Faculty of Mathematical Studies, University of Southampton, UK. 